\title{
Boundary conditions and amplitude ratios for finite-size corrections of a one-dimensional quantum spin model
}

\author{
N. Sh. Izmailian ${ }^{1,2,3,4, *}$ and Chin-Kun $\mathrm{Hu}^{1,5,+}$ \\ ${ }^{1}$ Institute of Physics, Academia Sinica, Taipei 11529, Taiwan \\ ${ }^{2}$ Yerevan Physics Institute, Alikhanian Br. 2, 375036 Yerevan, Armenia \\ 3 International Center for Advanced Study, Yerevan State University, \\ 1 Alex Manoogian St., Yerevan, 375025, Armenia \\ ${ }^{4}$ National Center of Theoretical Sciences at Taipei, Physics Division, \\ National Taiwan University, Taipei 10617, Taiwan and \\ ${ }^{5}$ Center for Nonlinear and Complex Systems and Department of Physics, \\ Chung-Yuan Christian University, Chungli 320, Taiwan
}

(Dated: October 29, 2018)

\begin{abstract}
We study the influence of boundary conditions on the finite-size corrections of a one-dimensional (1D) quantum spin model by exact and perturbative theoretic calculations. We obtain two new infinite sets of universal amplitude ratios for the finite-size correction terms of the 1D quantum spin model of $N$ sites with free and antiperiodic boundary conditions. The results for the lowest two orders are in perfect agreement with a perturbative conformal field theory scenario proposed by Cardy [Nucl. Phys. B 270, 186 (1986)].

PACS numbers: $05.50 .+\mathrm{q}, 05.70 . J \mathrm{k}, 11.25 . \mathrm{Hf}$
\end{abstract}




\section{INTRODUCTION}

Universality [3-9], finite-size scaling [4-16], and finite-size corrections [17-29] for critical lattice systems have attracted much attention in recent decades. It has been found that critical systems can be classified into different universality classes so that the systems in the same class have the same set of critical exponents, whose values depends only on the global properties of the system such as spacial dimensions, number of components of the order parameter, the range of interaction, and the symmetry group [ 30 . The hypothesis of universality has much stronger implications and it is possible to show that models belonging to the same universality class also share the same set of universal finite-size scaling functions (UFSSFs) and amplitude ratios [6 13, 21, 30, 31], whose values are independent of the microscopic structure of interactions. However, using a histogram Monte carlo simulation method [32], Hu, et al. confirmed for percolation the well-established dependence of finitesize scaling functions on boundary conditions and on the shape of the domains of the systems [10, 11, 14 16]. Based on exact partition functions of the Ising model on square, triangular, and honeycomb lattices [33], $\mathrm{Wu}, \mathrm{Hu}$ and Izmailian have found similar results for the Ising model [34].

In 1967-1969 Ferdinand and Fisher [17] calculated exact finite-size corrections (FSCs) for the free energy and its derivatives of the dimer and Ising models. In 1997, Ziff, Finch and Adamchik [18] used Monte Carlo simulations to calculate FSCs for cluster numbers of two-dimensional random percolation models. In 2002, Caselle, et al. [19] used conformal field theory to study correction terms for the free energy and its derivatives of the Ising model. Based on the connections between lattice phase transition models and correlated percolation models [35], in $1999 \mathrm{Hu}$, et al. [20] calculated FSCs for cluster numbers of the $q$-state Potts model for $q$ being 1, 2, 3 and 4. In such studies, they extended the calculations of Ferdinand and Fisher for the Ising model on torus [17] to higher orders. Such studies inspire further research on FSCs for the Ising [21] and the dimer models.

Based on Kronecker's double series and exact asymptotic expansions for exact partition functions [22], exact finite-size corrections for the Ising model [22 25] and the dimer model [26 29] on planar lattices with various boundary conditions have been obtained. It has been found that such correction terms also depend on the boundary conditions. In [21], we have found exact universal amplitude ratios for finite-size corrections of the two-dimensional 
Ising model on square, plane triangular and honeycomb lattices and a quantum spin chain on lattices with periodic boundary conditions. It is of interest to know how universal amplitude ratios depend on boundary conditions. In the present paper, we will address this question for a one dimensional quantum spin model with a varying parameter $\gamma$. We have calculated exact amplitude ratios for the quantum spin model with different boundary conditions and found that such ratios depend on boundary conditions and are independent of $\gamma$.

The quantitative description of the universality classes of critical behavior is the main goal of quantum field theory when it is applied to statistical mechanics. In principle, the universal quantities can be computed from the quantum field theory encoding the fundamental symmetries of the system.

The universality class can describe the critical behavior of many physical systems, which undergo a second order phase transition. The criteria by which the critical systems can be classified into different universality classes is a problem of much academic interest. Twodimensional (2D) critical systems are parameterized by the conformal anomaly $c$ which is the central charge in the Virasoro algebra [36 38]. The conformal anomaly $c$ can be obtained directly from the finite size corrections to the free energy for a 2D classical system on infinitely long but finitely wide strip at a conformal invariant critical point.

The asymptotic finite-size scaling behavior of the critical free energy $\left(f_{N}\right)$ and the inverse correlation lengths $\left(\xi_{i}^{-1}\right)$ of an infinitely long $2 \mathrm{D}$ strip of finite width $N$ at criticality has the form [39, 40]

$$
\begin{gathered}
\lim _{N \rightarrow \infty} N^{2}\left(f_{N}-f_{\infty}\right)-2 N f_{\text {surf }}=A, \\
\lim _{N \rightarrow \infty} N \xi_{i}^{-1}=D_{i},
\end{gathered}
$$

where $f_{\infty}$ is the bulk free energy, $f_{\text {surf }}$ is the surface free energy and $A$ and $D_{i}$ are the universal constants, but may depend on the boundary conditions (BCs). In some 2D geometries, the values of $A$ and $D_{i}$ are known [39-41], to be related to the conformal anomaly number $(c)$, the conformal weight of the ground state $(\Delta)$, and the scaling dimension of the $i$-th scaling field $\left(x_{i}\right)$ of the theory

$$
A=4 \pi\left(\frac{c}{24}-\Delta\right), \quad D_{i}=2 \pi x_{i}
$$

for periodic or antiperiodic BCs and

$$
A=\pi\left(\frac{c}{24}-\Delta\right), \quad D_{i}=\pi x_{i}
$$


for free BCs. For the 2D Ising model, we have $c=1 / 2$. The conformal weight of the ground state $\Delta$, and the scaling dimension $x_{i}$ are the universal quantities which depends on the BCs: for periodic BCs $\left(\Delta=0, x_{1}=1 / 8, x_{2}=1\right)$, for antiperiodic BCs $\left(\Delta=1 / 16, x_{1}=\right.$ $\left.3 / 8, x_{2}=1\right)$, and for free $\operatorname{BCs}\left(\Delta=0, x_{1}=1 / 2, x_{2}=2\right)$.

Quite recently, Izmailian and $\mathrm{Hu}$ [21] studied the finite size correction terms for the free energy per spin and the inverse correlation lengths of critical $2 \mathrm{D}$ Ising models on $N \times \infty$ lattice and 1D quantum Ising chain with periodic BCs. They obtained analytic expressions for the finite-size correction coefficients $a_{k}, b_{k}$ and $c_{k}$ in the expansions

$$
\begin{aligned}
N\left[f(N)-f_{\infty}\right] & =\sum_{k=1}^{\infty} \frac{a_{k}}{N^{2 k-1}}, \\
\xi_{s}^{-1}(N) & =\sum_{k=1}^{\infty} \frac{b_{k}}{N^{2 k-1}}, \\
\xi_{e}^{-1}(N) & =\sum_{k=1}^{\infty} \frac{c_{k}}{N^{2 k-1}},
\end{aligned}
$$

and find that although the finite-size correction coefficients $a_{k}, b_{k}$ and $c_{k}$ are not universal, the amplitude ratios for the coefficients of these series are universal and given by

$$
\begin{aligned}
& r_{s}(k)=\frac{b_{k}}{a_{k}}=\frac{2^{2 k}-1}{2^{2 k-1}-1}, \\
& r_{e}(k)=\frac{c_{k}}{a_{k}}=\frac{4 k}{\left(2^{2 k-1}-1\right) B_{2 k}},
\end{aligned}
$$

where $B_{n}$ is the $n$-th Bernoulli number $\left(B_{2}=1 / 6, B_{4}=-1 / 30, \ldots\right)$.

In the next section of the present paper we will present exact calculations for a set of universal amplitude ratios for the 1D quantum spin model [42], which is the quantum version of the classical 2D Ising model, with free and antiperiodic BCs. We obtain analytic equations for $a_{k}, b_{k}$ and $c_{k}$ in the expansions given by Eqs. (5), (66) and (77) and find that universal amplitude ratios for the 1D quantum Ising model with antiperiodic boundary condition are given by

$$
\begin{aligned}
& r_{s}(k)=\frac{b_{k}}{a_{k}}=\frac{\left(2^{2 k}-1\right) B_{2 k}-2 k}{2^{2 k-1} B_{2 k}} \\
& r_{e}(k)=\frac{c_{k}}{a_{k}}=-\frac{2 k}{B_{2 k}}
\end{aligned}
$$


while for free BCs we obtain

$$
\begin{aligned}
& r_{s}(k)=\frac{b_{k}}{a_{k}}=\frac{4 k}{\left(2^{2 k-1}-1\right) B_{2 k}}, \\
& r_{e}(k)=\frac{c_{k}}{a_{k}}=\frac{4 k\left(3^{2 k-1}+1\right)}{\left(2^{2 k-1}-1\right) B_{2 k}} .
\end{aligned}
$$

As far as we know, no previous RG arguments, analytic calculations, or numerical studies predict the existence of this whole set of universal amplitude ratios.

\section{ONE-DIMENSIONAL QUANTUM SPIN CHAIN}

The 1D quantum spin chain, the hamiltonian limit of the classical 2D Ising model (see [43] and references therein), belongs to the most frequently investigated and best understood systems in statistical physics. We will calculate universal amplitude ratios for this systems with anti-periodic boundary conditions and free boundary conditions.

\section{A. Anti-periodic boundary conditions}

Let us first consider the antiperiodic BCs. On a chain with $N$ sites the Hamiltonian is given by

$$
H=-\frac{\lambda}{2 \gamma} \sum_{n=1}^{N} \sigma_{n}^{z}-\frac{1}{4 \gamma} \sum_{n=1}^{N}\left[(1+\gamma) \sigma_{n+1}^{x} \sigma_{n}^{x}+(1-\gamma) \sigma_{n+1}^{y} \sigma_{n}^{y}\right],
$$

which was exactly solved by Katsura [42]. Here $\sigma^{x}, \sigma^{y}$ and $\sigma^{z}$ are the Pauli spin matrices. The antiperiodic BCs imposed on Eq. (14) are

$$
\sigma_{N+1}^{x}=-\sigma_{1}^{x} \quad \text { and } \quad \sigma_{N+1}^{y}=-\sigma_{1}^{y} .
$$

The phase diagram is well known [44]. For all $\gamma(0<\gamma \leq 1)$, there is a critical point at $\lambda_{c}=1$, which falls into the 2D Ising universality class. For $\gamma=1$ it is also called 1D transverse Ising model. Thus, by the introduction of a parameter $\gamma$ we could study different models in the same universality class. The Hamiltonian of Eq. (14) can be diagonalized by a Jordan-Wigner transformation as

$$
H=\sum_{k} \Lambda_{k}\left(\eta_{k}^{\dagger} \eta_{k}-1 / 2\right)
$$


where $\eta_{k}^{\dagger}, \eta_{k}$ are fermionic creation and annihilation operators and

$$
\Lambda_{k}=\sqrt{(\cos k-\lambda)^{2} / \gamma^{2}+\sin ^{2} k},
$$

is the lattice dispersion relation. At the critical point $\lambda_{c}=1$ one then obtains $\Lambda_{k}=2 \psi\left(\frac{k \pi}{2 N}\right)$. Here

$$
\psi(x)=\sqrt{\sin ^{2}(x)-\frac{\gamma^{2}-1}{\gamma^{2}} \sin ^{4}(x)} .
$$

The critical ground-state energy, $E_{0}^{(A)}$, corresponds to the antiperiodic BC has the value [45]

$$
E_{0}^{(A)}=-\sum_{m=0}^{N-1} \psi\left(\frac{\pi m}{N}\right)=-\sum_{m=0}^{N-1} \sqrt{\sin ^{2} \frac{\pi m}{N}-\frac{\gamma^{2}-1}{\gamma^{2}} \sin ^{4} \frac{\pi m}{N}}
$$

The energy gaps $\Delta_{s}^{(A)}$ and $\Delta_{e}^{(A)}$ are given by

$$
\begin{aligned}
& \Delta_{s}^{(A)}=2 \psi\left(\frac{\pi}{2 N}\right)+\sum_{m=0}^{N-1}\left[\psi\left(\frac{\pi m}{N}\right)-\psi\left(\frac{2 m+1}{2 N} \pi\right)\right], \\
& \Delta_{e}^{(A)}=2 \psi\left(\frac{\pi}{N}\right)=2 \sqrt{\sin ^{2} \frac{\pi}{N}-\frac{\gamma^{2}-1}{\gamma^{2}} \sin ^{4} \frac{\pi}{N}}
\end{aligned}
$$

Note, that the ground state energy $E_{0}$, the first energy gap $\left(E_{1}-E_{0} \equiv \Delta_{s}\right)$ and the second energy gap $\left(E_{2}-E_{0} \equiv \Delta_{e}\right)$ of a quantum spin chain are, respectively, the quantum analogies of the free energy $f(N)$, inverse spin-spin correlation length $\xi_{s}^{-1}(N)$, and inverse energy-energy correlation length $\xi_{e}^{-1}(N)$ for the Ising model; that is,

$$
N f(N) \Leftrightarrow-E_{0}, \xi_{s}^{-1}(N) \Leftrightarrow \Delta_{s} \text {, and } \xi_{e}^{-1}(N) \Leftrightarrow \Delta_{e} .
$$

To write $E_{0}^{(A)}, \Delta_{s}^{(A)}$, and $\Delta_{e}^{(A)}$ in the form of Eqs. (5), (6), and (7), we must evaluate Eqs. (18), (19), and (20) asymptotically. These sums can be handled by using the EulerMaclaurin summation formula [46]. Suppose that $F(x)$ together with its derivatives is continuous within the interval $(a, b)$. Then the general Euler-Maclaurin summation formula states

$$
\sum_{n=0}^{N-1} F(a+n h+\alpha h)=\frac{1}{h} \int_{a}^{b} F(\tau) \mathrm{d} \tau+\sum_{k=1}^{\infty} \frac{h^{k-1}}{k !} \mathrm{B}_{k}(\alpha)\left(F^{(k-1)}(b)-F^{(k-1)}(a)\right)
$$

where $0 \leq \alpha \leq 1, h=(b-a) / N$ and $\mathrm{B}_{k}(\alpha)$ are so-called Bernoulli polynomials defined in terms of the Bernoulli numbers $B_{p}$ by

$$
\mathrm{B}_{k}(\alpha)=\sum_{p=0}^{k} \mathrm{~B}_{p} \frac{k !}{(k-p) ! p !} \alpha^{k-p}
$$


Indeed, $B_{n}(0)=B_{n}$. Bernoulli polynomials satisfy the identity:

$$
B_{n}(1 / 2)=\left(2^{1-n}-1\right) B_{n}
$$

By expanding the exact solution of Eq. (14), Henkel [45] has obtained several finite-size correction terms to the ground-state energy $E_{0}$ and to the first energy gap $E_{1}-E_{0}$. We have extended the calculations to arbitrary order and found that

$$
\begin{aligned}
E_{0}^{(A)} & +N \alpha_{0}=\sum_{k=1}^{\infty} \frac{2 B_{2 k}}{(2 k) !}\left(\frac{\pi}{N}\right)^{2 k-1} \psi^{(2 k-1)} \\
& =\frac{\pi}{6 N}-\frac{1}{15}\left(\frac{1}{\gamma^{2}}-\frac{4}{3}\right)\left(\frac{\pi}{2 N}\right)^{3}+\ldots, \\
\Delta_{s}^{(A)} & =\sum_{k=1}^{\infty} \frac{4 k-2 B_{2 k}\left(2^{2 k}-1\right)}{(2 k) !}\left(\frac{\pi}{2 N}\right)^{2 k-1} \psi^{(2 k-1)} \\
& =\frac{3 \pi}{4 N}+\frac{9}{8}\left(\frac{1}{\gamma^{2}}-\frac{4}{3}\right)\left(\frac{\pi}{2 N}\right)^{3}+\ldots, \\
\Delta_{e}^{(A)} & =\sum_{k=1}^{\infty} \frac{4 k}{(2 k) !}\left(\frac{\pi}{N}\right)^{2 k-1} \psi^{(2 k-1)} \\
& =\frac{2 \pi}{N}+\left(\frac{1}{\gamma^{2}}-\frac{4}{3}\right)\left(\frac{\pi}{N}\right)^{3}+\ldots,
\end{aligned}
$$

where $\psi^{(2 k-1)}=\left(d^{2 k-1} \psi(x) / d x^{2 k-1}\right)_{x=0}$ and $\alpha_{0}$ is an non-universal number

$$
\alpha_{0}=\frac{1}{\pi} \int_{0}^{\pi} \psi(x) d x=\left[1+\arccos \gamma /\left(\gamma \sqrt{1-\gamma^{2}}\right)\right] / \pi
$$

The ratios of the amplitudes of the $N^{-(2 k-1)}$ correction terms in the spin-spin correlation length, energy-energy correlation length and the free energy expansion, i.e. $b_{k} / a_{k}$ and $c_{k} / a_{k}$, are $\gamma$-independent and are given by Eqs. (10) and (11), respectively, thus confirming the universality of this ratios.

\section{B. Free boundary conditions}

Let us now consider the transverse Ising model on a 1D lattice of $N$ sites with free BC and with the Hamiltonian

$$
H=-\lambda \sum_{n=1}^{N} \sigma_{n}^{z}-2 \sum_{n=1}^{N-1} \sigma_{n}^{x} \sigma_{n+1}^{x}
$$


In the limit $N \rightarrow \infty$, the ground state is singular at $\lambda=1$. The Ising chain in a transverse field has been studied in great detail (see e.g. [42, 47]). The Hamiltonian of Eq. (28) may be re-expressed in the diagonal form

$$
H=\sum_{k} \bar{\Lambda}_{k}\left(\eta_{k}^{\dagger} \eta_{k}-1 / 2\right)
$$

with dispersion relation

$$
\bar{\Lambda}_{k}=\sqrt{(\lambda-1)^{2}+4 \lambda \sin ^{2}(k / 2)} .
$$

For free BCs one finds that the allowed values of $k$ are determined by the secular equation [47]

$$
\lambda^{-1}=\sin [(N+1) k] / \sin (N k)
$$

At the critical field $\lambda_{c}=1$, the secular equation reduced to

$$
\tan (k N)=\cot (k / 2)
$$

so that

$$
k=\frac{(2 m+1) \pi}{2 N+1}, \quad m=0,1, \ldots, N-1
$$

and for the dispersion relation one then obtain $\bar{\Lambda}_{k}=2 \varphi\left(\frac{(k+1 / 2) \pi}{2 N+1}\right)$, where

$$
\varphi(x)=\sin (x)
$$

The ground-state energy $E_{0}^{(F)}$, the energy gaps $\Delta_{s}^{(F)}$ and $\Delta_{e}^{(F)}$ are given by [47]

$$
\begin{aligned}
& E_{0}^{(F)}=-\sum_{m=0}^{N-1} \varphi\left(\frac{m+\frac{1}{2}}{2 N+1} \pi\right)=\frac{1}{2}\left(1-\operatorname{cosec} \frac{\pi}{4 N+2}\right) \\
& \Delta_{s}^{(F)}=2 \varphi\left(\frac{\pi}{4 N+2}\right)=2 \sin \frac{\pi}{2(2 N+1)} \\
& \Delta_{e}^{(F)}=2\left(\sin \frac{\pi}{2(2 N+1)}+\sin \frac{3 \pi}{2(2 N+1)}\right)
\end{aligned}
$$


The asymptotic expansion of these quantities can be written in the following form

$$
\begin{aligned}
E_{0}^{(F)} & +N \alpha_{0}=\sum_{k=1}^{\infty} \frac{B_{2 k}\left(1-2^{2 k-1}\right.}{(2 k) !}\left(\frac{\pi}{4 N+2}\right)^{2 k-1} \varphi^{(2 k-1)} \\
& =-\frac{\pi}{24(2 N+1)}-\frac{7}{90}\left(\frac{\pi}{8 N+4}\right)^{3}+\ldots, \\
\Delta_{s}^{(F)} & =\sum_{k=1}^{\infty} \frac{2}{(2 k-1) !}\left(\frac{\pi}{4 N+2}\right)^{2 k-1} \varphi^{(2 k-1)} \\
& =\frac{\pi}{2 N+1}-\frac{1}{3}\left(\frac{\pi}{4 N+2}\right)^{3}+\ldots, \\
\Delta_{e}^{(F)} & =\sum_{k=1}^{\infty} \frac{2\left(3^{2 k-1}+1\right)}{(2 k-1) !}\left(\frac{\pi}{4 N+2}\right)^{2 k-1} \varphi^{(2 k-1)} \\
& =\frac{4 \pi}{2 N+1}-\frac{28}{3}\left(\frac{\pi}{4 N+2}\right)^{3}+\ldots,
\end{aligned}
$$

where, $\varphi^{(2 k-1)}=\left(d^{2 k-1} \varphi(x) / d x^{2 k-1}\right)_{x=0}, \varphi(x)=\sin x$, and $\alpha_{0}=\frac{1}{\pi} \int_{0}^{\pi} \varphi(x) d x=2 / \pi$.

Equations (37), (38), and (39) imply that the ratios of the amplitudes of the $N^{-(2 k-1)}$ correction terms in the spin-spin correlation length, the energy-energy correlation lengths, and the free energy expansion, i.e. $b_{k} / a_{k}$ and $c_{k} / a_{k}$, should not depend in detail on the dispersion relation as given by Eqs. (12) and (13).

The leading terms of Eqs. (24) - (26), (37) - (39) are consistent with Eqs. (11) - (41), i.e. $a_{1}, b_{1}$ and $c_{1}$ are universal. Equations (11) and (2) implies immediately that their ratio is also universal, namely $r_{s}(1)=D_{1} / A$ and $r_{e}(1)=D_{2} / A$, which is consistent with Eqs. (8), (10), and Eq. (12) for the case $k=1$

$$
r_{s}(1)=\frac{D_{1}}{A}= \begin{cases}3 & \text { for periodic BC } \\ -9 / 2 & \text { for antiperiodic BC } \\ 24 & \text { for free BC }\end{cases}
$$

and with Eqs. (9), (11), and Eq. (13) for the case $k=1$

$$
r_{e}(1)=\frac{D_{2}}{A}= \begin{cases}24 & \text { for periodic BC } \\ -12 & \text { for antiperiodic BC } \\ 96 & \text { for free BC }\end{cases}
$$




\section{PERTURBATIVE CONFORMAL FIELD THEORY}

The finite-size corrections to Eqs. (11) and (21) can be calculated by the means of a perturbative conformal field theory [48, 49]. In general, any lattice Hamiltonian will contain correction terms to the critical Hamiltonian $H_{c}$

$$
H=H_{c}+\sum_{p} g_{p} \int_{-N / 2}^{N / 2} \phi_{p}(v) d v,
$$

where $g_{p}$ is a non-universal constant and $\phi_{p}(v)$ is a perturbative conformal field. Below we will consider the case with only one perturbative conformal field, say $\phi_{l}(v)$. Then the eigenvalues of $H$ are

$$
E_{n}=E_{n, c}+g_{l} \int_{-N / 2}^{N / 2}<n\left|\phi_{l}(v)\right| n>d v+\ldots,
$$

where $E_{n, c}$ are the critical eigenvalues of $H$. The matrix element $\left\langle n\left|\phi_{l}(v)\right| n\right\rangle$ can be computed in terms of the universal structure constants $\left(C_{n l n}\right)$ of the operator product expansion [48]: $\left\langle n\left|\phi_{l}(v)\right| n\right\rangle=(2 \pi / N)^{x_{l}} C_{n l n}$, where $x_{l}$ is the scaling dimension of the conformal field $\phi_{l}(v)$. The energy gaps $\left(\Delta_{n}=E_{n}-E_{0}\right)$ and the ground-state energy $\left(E_{0}\right)$ can be written as

$$
\begin{aligned}
& \Delta_{n}=\frac{2 \pi}{N} x_{n}+2 \pi g_{l}\left(C_{n l n}-C_{0 l 0}\right)\left(\frac{2 \pi}{N}\right)^{x_{l}-1}+\ldots, \\
& E_{0}=E_{0, c}+2 \pi g_{l} C_{0 l 0}\left(\frac{2 \pi}{N}\right)^{x_{l}-1}+\ldots
\end{aligned}
$$

For the 2D Ising model, one finds [21] that at least two (and probably infinitely many) perturbative conformal fields are necessary to generate all finite-size corrections terms. Nevertheless, the leading finite-size corrections $\left(1 / N^{3}\right)$ can be described by the Hamiltonian given by Eq. (42) with a single perturbative conformal field $\phi_{l}(v)=L_{-2}^{2}(v)+\bar{L}_{-2}^{2}(v)$ with scaling dimension $x_{l}=4$ [50].

In order to obtain the corrections we need the matrix elements $\left\langle n\left|L_{-2}^{2}(v)+\bar{L}_{-2}^{2}(v)\right| n>\right.$, which have already been computed by Reinicke [51]:

$$
\begin{aligned}
<\Delta+r\left|L_{-2}^{2}\right| \Delta+r> & =\left(\frac{2 \pi}{N}\right)^{4}\left[\frac{49}{11520}+(\Delta+r)\left(\Delta-\frac{5}{24}+\frac{r(2 \Delta+r)(5 \Delta+1)}{(\Delta+1)(2 \Delta+1)}\right)\right] \\
<r\left|L_{-2}^{2}\right| r> & =\left(\frac{2 \pi}{N}\right)^{4}\left[\frac{49}{11520}+\frac{49}{120} r\left(2 r^{2}-3\right)\right]
\end{aligned}
$$

The universal structure constants $C_{2 l 2}, C_{1 l 1}$ and $C_{0 l 0}$ can be obtained from the matrix element

$$
<n\left|L_{-2}^{2}(v)+\bar{L}_{-2}^{2}(v)\right| n>=(2 \pi / N)^{x_{l}} C_{n l n},
$$


where $x_{l}=4$ is the scaling dimension of the conformal field $L_{-2}^{2}(v)+\bar{L}_{-2}^{2}(v)$.

At the critical point $\lambda_{c}=1$ the spectra of the Hamiltonian (14) with periodic and antiperiodic BC are built by the irreducible representation $\Delta, \bar{\Delta}$ of two commuting Virasoro algebras $L_{n}$ and $\bar{L}_{n}$ with central charge $c=\frac{1}{2}$ [41]. We denote by $\Delta$ the highest weight, and by $\Delta+r$, the $r$-th level having degeneracy $d(\Delta, r)$ of one irreducible representation of the Virasoro algebra. A state will be labelled by $|n>\sim| \Delta+r, \bar{\Delta}+\bar{r}>$. The possible values of $\Delta, \bar{\Delta}$ are $(0,0),\left(\frac{1}{2}, \frac{1}{2}\right),\left(\frac{1}{16}, \frac{1}{16}\right)$ for periodic and $\left(0, \frac{1}{2}\right),\left(\frac{1}{2}, 0\right),\left(\frac{1}{16}, \frac{1}{16}\right)$ for antiperiodic BCs. In the case of free BCs the spectra can be understood in terms of irreducible representations $\Delta$ of a single Virasoro algebra with possible values of $\Delta$ are $0, \frac{1}{2}$.

The ground state $\mid 0>$, first excited state $\mid 1>$, and second excited state $\mid 2>$ depends on the boundary conditions and given by [48, 52]:

$$
\begin{aligned}
& |0>=| \Delta=0, r=0 ; \bar{\Delta}=0, \bar{r}=0>, \\
& |1>=| \Delta=\frac{1}{16}, r=0 ; \bar{\Delta}=\frac{1}{16}, \bar{r}=0>, \\
& |2>=| \Delta=\frac{1}{2}, r=0 ; \bar{\Delta}=\frac{1}{2}, r=0>,
\end{aligned}
$$

for periodic BCs;

$$
\begin{aligned}
& |0>=| \Delta=\frac{1}{16}, r=0 ; \bar{\Delta}=\frac{1}{16}, \bar{r}=0>, \\
& |1>=| \Delta=0, r=0 ; \bar{\Delta}=\frac{1}{2}, \bar{r}=0>, \\
& |2>=| \Delta=\frac{1}{16}, r=0 ; \bar{\Delta}=\frac{1}{16}, \bar{r}=1>,
\end{aligned}
$$

for antiperiodic BCs; and

$$
\begin{aligned}
& |0>=| \Delta=0, r=0>, \\
& |1>=| \Delta=\frac{1}{2}, r=0>, \\
& |2>=| \Delta=0, r=2>
\end{aligned}
$$

for free BCs.

After reaching this point, one can easily compute the universal structure constants $C_{0 l 0}$, $C_{1 l 1}$ and $C_{2 l 2}$ for all three boundary conditions. The values of $C_{0 l 0}, C_{1 l 1}, C_{2 l 2}$ can be obtained from Eqs. (46) - (57) and given by:

$$
C_{0 l 0}=49 / 5760, \quad C_{1 l 1}=-7 / 720, \quad C_{2 l 2}=1729 / 5760,
$$


for periodic BCs;

$$
C_{0 l 0}=-7 / 720, \quad C_{1 l 1}=889 / 5760, \quad C_{2 l 2}=833 / 720
$$

for antiperiodic BCs; and

$$
C_{0 l 0}=49 / 11520, \quad C_{1 l 1}=1729 / 11520, \quad C_{2 l 2}=47089 / 11520,
$$

for free BCs. Equations (44) and (45) implies that the ratios of first-order corrections amplitudes for $\left(\Delta_{n}\right)$ and $\left(-E_{0}\right)$ is universal and equal to $\left(C_{0 l 0}-C_{n l n}\right) / C_{0 l 0}$, which is consistent with Eqs. (8), (10), and Eq. (12) for the case $k=2$

$$
r_{s}(2)=\frac{C_{0 l 0}-C_{1 l 1}}{C_{0 l 0}}=\left\{\begin{array}{lc}
15 / 7 & \text { for periodic BCs } \\
135 / 8 & \text { for antiperiodic BCs } \\
-240 / 7 & \text { for free BCs }
\end{array}\right.
$$

and with Eqs. (9), (11), and Eq. (13) for the case $k=2$

$$
r_{e}(2)=\frac{C_{0 l 0}-C_{2 l 2}}{C_{0 l 0}}= \begin{cases}-240 / 7 & \text { for periodic BCs, } \\ 120 & \text { for antiperiodic BCs } \\ -960 & \text { for free BCs. }\end{cases}
$$

\section{CONCLUSION}

In this paper we discuss the influence of the boundary conditions on the finite-size corrections of the 2D Ising model in the extreme anisotropic or quantum-Hamiltonian limit. We have calculated various universal amplitude ratios and find that such result are in perfect agreement with a perturbated conformal field theory scenario proposed by Cardy [48].

The results of this paper inspire several problems for further studies: (i) Further work has to be done to possibly evaluate exactly all finite-size correction terms from perturbative conformal field theory. (ii) Can one obtain from the perturbated conformal field theory the value of the universal amplitude ratios $\left(r_{s}(k)\right.$ and $\left.r_{e}(k)\right)$ for $k>2$ ? (iii) How do such amplitudes behave in other models, for example in the three-state Potts model? (iv) Our results also present new challenges to scientists working on numerical studies of critical phenomena. For example, it is of interest to present accurate numerical evidences about whether the Ising model on a two-dimensional lattice with crossing bonds has the same set of amplitude ratios. 


\section{ACKNOWLEDGEMENTS}

One of us (NSI) would like to thank A. Zamolodchikov for helpful comments and discussions. This work was supported by National Science Council of the Republic of China (Taiwan) under Grant No. NSC 96-2911-M 001-003-MY3 and National Center for Theoretical Sciences in Taiwan.

[*] Electronic address: izmailan@phys.sinica.edu.tw.

[+] Electronic address: huck@phys.sinica.edu.tw.

[3] H. E. Stanley, Introduction to Phase Transitions and Critical Phenomena (Oxford Univ. Press, New York, 1971).

[4] M. N. Barber, in: C. Domb and J. L. Lebowitz (Eds.), Phase Transition and Critical Phenomena, Vol. VIII, Academic Press, New York, 1983.

[5] M. E. Fisher, in Proc. 1970 E. Fermi Int. School of Physics, M. S. Green ed. (Academic, NY, 1971) Vol. 51, p. 1.

[6] V. Privman, M.E. Fisher, Phys. Rev. B 30 (1984) 322.

[7] V. Privman, ed., Finite-size Scaling and Numerical Simulation of Statistical Systems (World Scientific, Singapore, 1990).

[8] V. Privman, P.C. Hohenberg, A. Aharony, in: C. Domb and J. Lebowitz (Eds.), Phase Transition and Critical Phenomena, Vol. XIV, Academic Press, New York, 1991, p1.

[9] J. Zinn-Justin, Quantum Field Theory and Critical Phenomena, fourth ed., Clarendon, Oxford, 2001.

[10] C.-K. Hu, C.-Y. Lin, J.-A. Chen, Phys. Rev. Lett. 75 (1995) 193 and 75 (1995) 2786(E), Physica A 221 (1995) 80; C.-K. Hu, C.-Y. Lin, Phys. Rev. Lett. 77 (1996) 8; C.-K. Hu, F.-G. Wang, J. Korean Physical Soc. 31 (1997) S271; H. P. Hsu, S. C. Lin, C.-K. Hu, Phys. Rev. E 64 (2001) 016127; H. Watanabe, S. Yukawa, N. Ito, C.-K. Hu, C.Y. Lin, W.J. Ma, J. Phys. Soc. Japan. 70 (2001) 1537.

[11] C.-Y. Lin, C.-K. Hu, Phys. Rev. E, 58 (1998) 1521; C.-Y. Lin, C.-K. Hu, J.-A. Chen, J. Phys. A: Math. Gen. 31 (1998) L111.

[12] Y. Okabe, M. Kikuchi, International J. Modern Phys. C 7, 287 (1996); F. G. Wang, C.-K. 
Hu, Phys. Rev. E 56 (1997) 2310.

[13] Y. Okabe, K. Kaneda, M. Kikuchi, C.-K. Hu, Phys. Rev. E 59 (1999) 1585; Y. Tomita, Y. Okabe, C.-K. Hu, ibid. 60 (1999) 2716; C.-K. Hu, J.-A. Chen, C.-Y. Lin, Physica A 266 (1999) 27 .

[14] H. Watanabe, S. Yukawa, N. Ito, C.-K. Hu, Phys. Rev. Lett. 93 (2004) 19601. This paper contains some typos, see Ref. [15] for details; see also G. Pruessner, N. R. Moloney, Phys. Rev. Lett. 95 (2005) 258901.

[15] H. Watanabe, C.-K. Hu, Phys. Rev. Lett. 95 (2005) 258902; Phys. Rev. E, arXiv: 0809.3636 [cond-mat.stat-mech].

[16] C.-K. Hu, J. Phys. A: Math. Gen. 27 (1994) L813; C.-K. Hu, J.-A. Chen, ibid. 28 (1995) L73; C.-K. Hu, Phys. Rev. Lett. 76 (1996) 3875.

[17] A. E. Ferdinand, J. Math. Phys. 8 (1967) 2332; A. E. Ferdinand and M. E. Fisher, Phys. Rev. 185 (1969) 832.

[18] R. M. Ziff, S. R. Finch and V. S. Adamchik, Phys Rev. Lett. 79 (1997) 3447.

[19] M. Caselle, M. Hasenbusch, A. Pelissetto, E. Vivari, J. Phys. A 35 (2002) 4861.

[20] C.-K. Hu, J.-A. Chen, N.S. Izmailian, P. Kleban, Phys. Rev. E 60 (1999) 6491.

[21] N.Sh. Izmailian, C.-K. Hu, Phys. Rev. Lett. 86 (2001) 5160.

[22] E. Ivashkevich, N.Sh. Izmailian, C.-K. Hu, J. Phys. A 35 (2002) 5543.

[23] J. Salas, J. Phys. A: Math. Gen. 34 (2001) 1311; 35 (2002) 1833; W. Janke, R. Kenna, Phys. Rev. B 65 (2002) 064110.

[24] Y. Okabe and N. Kawashima, Phys. Rev. E 64 (2001) 035103(R).

[25] N.Sh. Izmailian, C.-K. Hu, Phys. Rev. E 65 (2002) 036103; N.Sh. Izmailian, K.B. Oganesyan, C.-K. Hu, Phys. Rev. E 65 (2002) 056132; N. Sh. Izmailian, C.-K. Hu, Phys. Rev. E 76 (2007) 041118 .

[26] N.Sh. Izmailian, K.B. Oganesyan, C.-K. Hu, Phys. Rev. E 67 (2003) 066114.

[27] N. Sh. Izmailian, V. B. Priezzhev, P. Ruelle, C.-K. Hu, Phys. Rev. Lett. 95 (2005) 260602.

[28] N. Sh. Izmailian , V. B. Priezzhev, P. Ruelle, SIGMA 3 (2007) 001.

[29] N. Sh. Izmailian, K. B. Oganesyan, M.-C. Wu, C.-K. Hu, Phys. Rev. E 73 (2006) 016128.

[30] G. Delfino, Phys. Lett. B 419 (1998) 291.

[31] G. Delfino, P. Grinza, Nucl. Phys. B 682 (2004) 521.

[32] C.-K. Hu, Phys. Rev. B 46 (1992) 6592; Phys. Rev. Lett. 69 (1992) 2739. 
[33] M.-C. Wu, C.-K. Hu, J. Phys. A: Math. Gen. 35 (2002) 5189.

[34] M.-C. Wu, C.-K. Hu, N.Sh. Izmailian, Phys. Rev. E 67 (2003) 065103(R).

[35] C.-K. Hu, Physica A 116 (1982) 265; ibid. 119 (1983) 609; J. Phys. A: Math. Gen. 16 (1983) L321; Phys. Rev. B 29 (1984) 5103; ibid. 29 (1984) 5109; C.-K. Hu and K.-S. Mak, ibid. 39 (1989) 2948; ibid. 42 (1990) 965; ibid. 40 (1989) 5007.

[36] A.A. Belavin, A.M. Polyakov, A.B. Zamolodchikov, Nucl. Phys. B241 (1984) 333.

[37] Vl.S. Dotsenko, V.A. Fateev, Nucl. Phys. B240 (1984) 312.

[38] Vl.S. Dotsenko, V.A. Fateev, Nucl. Phys. B251 (1985) 691.

[39] H.W.J. Blöte, J.L. Cardy, M.P. Nightingale, Phys. Rev. Lett. 56 (1986) 742.

[40] I. Affleck, Phys. Rev. Lett. 56 (1986) 746.

[41] J. Cardy, Nucl. Phys. B 275 (1986) 200.

[42] S. Katsura, Phys. Rev. 127 (1962) 1508.

[43] J. Kogut, Rev. Mod. Phys. 51 (1979) 659.

[44] E. Barouch, B. M. McCoy, Phys. Rev. A 3 (1971) 746.

[45] M. Henkel, J. Phys. A 20 (1987) 995.

[46] G.H. Hardy, Divergent Series, (Clarendon Press, Oxford, 1949).

[47] P. Pfeuty, Ann. Phys., NY 57 (1970) 79.

[48] J. Cardy, Nucl. Phys. B 270 (1986) 186.

[49] A.B. Zamolodchikov, Sov. J. Nucl. Phys. 46 (1987) 1090.

[50] For more details see M. Henkel, Conformal invariance and critical phenomena, (Springer Verlag, Heidelberg, 1999), Chap. 13.

[51] P. Reinicke, J. Phys. A 20 (1987) 5325.

[52] G. von Gehlen, V. Rittenberg, J. Phys. A 19 (1988) L631. 